\title{
Disulfiram induced hemoptysis: a case report
}

\author{
Ravishankar M.*, Rakshith N.
}

Department of Pharmacology, Adichunchanagiri Institute of Medical Sciences, B.G. Nagar, Mandya district, Karnataka, India

Received: 06 November 2015 Accepted: 18 December 2015

\section{*Correspondence to:}

Dr. Ravishankar M,

Email: ravipharmac@yahoo.com

Copyright: (C) the author(s), publisher and licensee Medip Academy. This is an open-access article distributed under the terms of the Creative Commons Attribution Non-Commercial License, which permits unrestricted non-commercial use, distribution, and reproduction in any medium, provided the original work is properly cited.

\begin{abstract}
Disulfiram (tetraethylthiuram disulfide [TETD]) has been used for more than 50 years as a deterrent to ethanol abuse in the management of alcoholism. Approximately 200,000 alcoholics take disulfiram, or Antabuse, regularly in the United States. Haemoptysis is the expectoration of blood originating from the lower respiratory tract. It is a common alarming symptom accounting for 10 to $15 \%$ of all pulmonary visits. Here, we present the case report of a 39 -year old male patient who came to the medicine OPD with complaints of coughing of blood in the early morning hours since one and a half months. During history taking, it was understood that the patient was administered disulfiram by his wife, without his knowledge to prevent him from consuming alcohol since one and a half months (the patient is a chronic alcoholic). There was no history of any other drug intake which could cause haemoptysis like anticoagulants, thrombolytics and non-steroidal anti-inflammatory drugs. Infections causing haemoptysis like tuberculosis, pneumonia and bronchitis were ruled out. Haemoptysis subsided once disulfiram was stopped. Thus, in this case, because of the temporal relationship between exposure to the drug and the onset of symptoms, disulfiram was considered as the most probable cause of haemoptysis. The adverse drug reaction was considered probable (score 5) according to the Naranjo adverse drug reaction probability scale.
\end{abstract}

Keywords: Disulfiram, Antabuse, Hemoptysis, Alcoholism, Aldehyde syndrome, Tuberculosis, Pneumonia, Bronchitis, Naranjo adverse drug reaction probability scale

\section{INTRODUCTION}

Disulfiram (tetraethylthiuram disulfide [TETD]) has been used for more than 50 years as a deterrent to ethanol abuse in the management of alcoholism. Approximately 200,000 alcoholics take disulfiram, or antabuse, regularly in the United States.

The first suggestion that disulfiram might be used in the treatment of alcoholism came in 1937 when an American physician noted that workers in the rubber industry who were exposed to TETD developed a reaction after drinking ethanol. A decade later two Danish researchers at the Royal Danish School of Pharmacy in Copenhagen made the same discovery. Jens Hald and Eric Jacobson were experimenting with disulfiram as a potential anthelminthic, and each took small doses to determine potential side effects in humans. Several days later, they attended a cocktail party and both became ill. They concluded that the facial flushing and tachycardia they experienced must be due to the disulfiram.

Soon thereafter, physicians began prescribing disulfiram as a deterrent to ethanol abuse. It has also been proposed as a deterrent to cocaine abuse, and several studies have suggested improved retention rates in treatment programs for cocaine-dependant individuals treated with disulfiram. A study found diminished "high" or "rush" after intravenous cocaine administration to healthy volunteers pretreated with disulfiram, with no change in cardiovascular parameters. ${ }^{1}$

The disulfiram-ethanol reaction (DER) is due to increased serum acetaldehyde concentrations generated by the metabolism of ethanol by alcohol dehydrogenase in the liver. Normally, this acetaldehyde is cleared rapidly by its metabolism to acetate via aldehyde dehydrogenase. ${ }^{2}$

Disulfiram blocks this enzyme, irreversibly inhibiting the oxidation of acetaldehyde and causing a marked increase in acetaldehyde concentrations after ethanol consumption. The discomfort associated with this syndrome is intended to serve as a negative stimulus, but the reaction may be severe enough to cause hypotension and death. 
Hemoptysis is the expectoration of blood originating from the lower respiratory tract. It is a common alarming symptom accounting for 10 to $15 \%$ of all pulmonary visits. $^{3-5}$

Hemoptysis is defined as the spitting of blood from the lungs or bronchial tubes as a result of pulmonary or bronchial hemorrhage. ${ }^{6}$

In the primary care setting, the most common causes of hemoptysis are acute and chronic bronchitis, pneumonia, tuberculosis, and lung cancer. ${ }^{7}$

Drug induced hemoptysis may occur due to exposure to anticoagulants, nonsteroidal anti-inflammatory drugs and thrombolytic agents. Till date there are no reports of hemoptysis due to disulfiram.

\section{CASE REPORT}

A 39-year old male patient came to the medicine OPD with complaints of coughing of blood in the early morning hours since one and a half months. He also complained of generalized weakness and pain in the peripheral joints of the upper and lower limbs since 1 day. Patient also complained of a difficulty in swallowing and is a known case of type 2 diabetes mellitus since 1 year, and hypertension was diagnosed 20 days back.

During the elicitation of the case history with the patient, it was also understood that the patient was given a drug by his wife, without his knowledge, to prevent him from consuming alcohol since one and a half months. The patient had a history of alcoholism for the past 23 years, during which he was consuming approximately $180 \mathrm{ml}$ of alcohol per day. The drug administered without the patient's knowledge was found to be disulfiram. As the patient was unaware of this scenario, every time he consumed alcohol, he developed uneasiness, difficulty in swallowing and coughing of blood in the early morning hours since one and a half months. But since one day, due to generalized weakness and distal joint pains, the patient came to the medicine OPD to seek medical attention.

Patient gives no history of any other drug intake or history of fever or any other illness. General physical examination was normal. Systemic examination revealed mild tenderness over the right and left lumbar and paraumbilical regions. Ultrasound examination revealed mild hepatomegaly and fatty changes.

The case was diagnosed as "Alcohol dependence syndrome and alcoholic liver disease". Disulfiram tablet was discontinued. Patient was treated accordingly, for the confirmed diagnosis. Ziehl-Neelsen staining was negative, sputum culture was negative.

Patient was put on syrup linctus codeine 2 tsp three times daily, Tab Glycomet (metformin) $500 \mathrm{mg}$ once daily, Tab Diapride (glimepiride) $1 \mathrm{mg}$ twice daily, Tab Voger (voglibose) once daily, Syrup Liv 52 three times daily, Tab Envas (enalapril) $2.5 \mathrm{mg}$ once daily, Tab Librium (chlordiazepoxide) $10 \mathrm{mg}$ three times daily, Capsules Becosules (Vitamin B complex with Vitamin C) once daily. Supportive measures were provided. Improvement was noted. Haemoptysis was not seen. Weakness and joint pain subsided. Rechallenge was not done.

\section{DISCUSSION}

In considering disulfiram toxicity, a distinction must be made between the clinical manifestations of a disulfiramethanol reaction (DER) and the toxic effects of disulfiram itself. Direct disulfiram toxicity may be further divided into acute poisoning versus chronic poisoning. The directly toxic effects of disulfiram include neurologic, cutaneous and hepatotoxic sequelae in addition to the disulfiram-ethanol reaction.

Disulfiram received US Food and Drug Administration (FDA) approval for use in the treatment of alcoholism in 1951. At that time, it was commonly prescribed in very high doses, up to $3,000 \mathrm{mg}$ a day in some cases. This resulted in a relatively high rate of extremely severe or fatal reactions. Today, much lower doses are used, and the incidence of disulfiram toxicity has waned.

Disulfiram is usually prescribed at an initial dose of 500 $\mathrm{mg} / \mathrm{d}$ for 1-2 weeks, followed by a maintenance dose of $125-500 \mathrm{mg} / \mathrm{d}$. Close monitoring of adverse reactions is required. Disulfiram use is associated with adverse reactions at a rate of approximately 1 per 200-2000 each year. Drowsiness is the most common side effect and occurs in up to $5 \%$ of patients. It generally resolves after 2 weeks of treatment. Other side effects include dyspnea, sweating, alteration of taste, vasodilation, impotence, amblyopia, dizziness, headache, ataxia, polyneuritis, psychosis and hypertension.

Acute disulfiram overdose is uncommon. In adults, clinical manifestations after acute overdose are rare with doses less than $3 \mathrm{~g}$. Ingestion of 10-30 g may be lethal. Toxicity in children has been reported after ingestion of $2.5 \mathrm{~g}$ of disulfiram. Symptoms of overdose in children are mostly neurologic.

Acetaldehyde syndrome may present with the following findings: ${ }^{8}$

Head, neck and chest flushing - histamine-induced vasodilation, throbbing headaches, nausea, vomiting, diarrhoea and abdominal pain, weakness, dizziness, confusion and anxiety, vertigo and ataxia, orthostatic hypotension, diaphoresis, palpitations and dysrhythmias, pruritus and refractory cyanosis.

Signs and symptoms of acute disulfiram overdose in adults and children include the following: Hypotension, tachycardia and dyspnea, abdominal pain, nausea and sulfur or garlic odour on breath, coma and seizures, 
Parkinson-like syndrome, polyneuropathy, hypersensitive hepatitis and hepatic failure and loss of developmental milestones.

In this scenario, disulfiram was administered to the patient without his knowledge. Primary causes of haemoptysis like tuberculosis, bronchitis and pneumonia were ruled out. There was also no history of thrombolytic, anticoagulant or non-steroidal antiinflammatory drug use. Thus, in this case, because of the temporal relationship between exposure to the drug and the onset of symptoms, disulfiram was considered as the most probable cause of haemoptysis. The adverse drug reaction was considered probable (score 5) according to the Naranjo adverse drug reaction probability scale. ${ }^{9}$

\section{CONCLUSION}

Disulfiram has been used for more than 50 years as a deterrent to ethanol abuse in the management of alcoholism. In this situation, it had been given to the patient without his knowledge. The temporal relationship between the exposure to disulfiram and the onset of haemoptysis suggests disulfiram to be the probable cause for the haemoptysis. The absence of other causes of haemoptysis like bronchitis, pneumonia and tuberculosis and exposure to haemoptysis causing drugs like anticoagulants, thrombolytics and non-steroidal antiinflammatory agents also goes in this favour. Till date there are no reports of haemoptysis due to disulfiram. The adverse drug reaction was considered probable (score 5) according to the Naranjo adverse drug reaction probability scale. ${ }^{9}$

\section{ACKNOWLEDGEMENTS}

We are grateful to the Department of Medicine, Adichunchanagiri Institute of Medical Sciences, B.G.Nagar.
Funding: No funding sources Conflict of interest: None declared

Ethical approval: Not required

\section{REFERENCES}

1. Baker JR, Jatlow P, McCance-Katz EF. Disulfiram effects on responses to intravenous cocaine administration. Drug Alcohol Depend. 2007;87(23):202-9.

2. Kong D, Kotraiah V. Modulation of aldehyde dehydrogenase activity affects ( \pm )-4-hydroxy-2Enonenal (HNE) toxicity and HNE-protein adduct levels in PC12 cell. J Mol Neurosci. 2012;47(3):595603.

3. O’Neil KM, Lazarus AA. Hemoptysis. Indications for bronchoscopy. Arch Intern Med. 1991;151:1714.

4. Pursel SE, Lindskog GE. Hemoptysis. A clinical evaluation of 105 patients examined consecutively on a thoracic surgical service. Am Rev Respir Dis. 1961;84:329-36.

5. Smiddy JF, Elliott RC. The evaluation of hemoptysis with fibreoptic bronchoscopy. Chest. 1973;64:15862.

6. Stedman TL. Stedman's medical dictionary. $27^{\text {th }}$ edition. Philadelphia: Lipincott Williams \& Wilkins; 2000.

7. Harrison TR, Braunwald E. Hemoptysis. In: Harrison's Principles of Internal Medicine. $15^{\text {th }}$ edition. New York: McGraw-Hill; 2001:203-6.

8. Valgini F, Viaggi C, Piro V, Pardini C, Gearace C, Scarselli M, Corsini GU. Acetaldehyde and parkinsonism: role of CYP450 2E1. Front Behav Neurosci. 2013;7:71.

9. Naranjo CA et al. A method for estimating the probability of adverse drug reactions. Clin Pharmacol Ther. 1981;30:239-45.

Cite this article as: Ravishankar M, Rakshith N. Disulfiram induced hemoptysis: a case report. Int $\mathbf{J}$ Basic Clin Pharmacol 2016;5:220-2. 